\title{
Le banni et l'ennemi. D'une technique policière de maintien de la tranquillité et de l'ordre publics
}

\section{Fabien Jobard}

\section{(2) OpenEdition \\ 1 Journals}

\section{Édition électronique}

URL : https://journals.openedition.org/conflits/571

DOI : $10.4000 /$ conflits. 571

ISSN : $1777-5345$

\section{Éditeur :}

CECLS - Centre d'études sur les conflits - Liberté et sécurité, L'Harmattan

\section{Édition imprimée}

Date de publication : 1 septembre 2001

ISBN : 2-7475-1118-9

ISSN : 1157-996X

\section{Référence électronique}

Fabien Jobard, «Le banni et l'ennemi. D'une technique policière de maintien de la tranquillité et de l'ordre publics », Cultures \& Conflits [En ligne], 43 | automne 2001, mis en ligne le 28 février 2003, consulté le 21 septembre 2021. URL : http://journals.openedition.org/conflits/571 ; DOI : https:// doi.org/10.4000/conflits.571

Ce document a été généré automatiquement le 21 septembre 2021.

Creative Commons License 


\title{
Le banni et l'ennemi. D'une technique policière de maintien de la tranquillité et de l'ordre publics
}

\author{
Fabien Jobard
}

\begin{abstract}
Les vagabonds sont pour les campagnes le fléau le plus terrible. [Ce sont des insectes voraces qui désolent journellement la subsistance des cultivateurs]. Ce sont, pour parler sans figure, des troupes d'ennemies répandues sur la surface $d u$ territoire, qui y vivent à discrétion comme dans un pays conquis et y lèvent de véritables contributions sous le titre d'aumônes ${ }^{1}$.

"Tout malfaiteur, attaquant le droit social, devient par ses forfaits, rebelle et traître à la patrie ; alors la conservation de l'État est incompatible avec la sienne ; il faut qu'un des deux périsse, et quand on fait périr le coupable, c'est moins comme citoyen que comme ennemi $»^{2}$.

«Mais l'ennemi aussi a son statut: il n'est pas un simple criminel » ${ }^{3}$.
\end{abstract}

«L'ennemi intérieur » est une figure, rien d'autre qu'une image. Il est l'un des termes que des institutions publiques, notamment répressives, emploient pour désigner tout à la fois leur objet et leur activité. Celle-ci consiste, en effet, à connaître ce qui est dans l'ombre et à l'amener à la lumière : " élucider un fait » (" porter un fait à la lumière ») suffit à en rendre compte, et ce non seulement en français, mais aussi en allemand, où l'on parle même de la « Aufklärung einer Straftat ». Le problème semble d'emblée saisi dans son énoncé : l'enquête de police, le travail de surveillance aux fins de répression du crime, aspirent d'emblée à se faire sciences sociales pratiques, sciences pratiques du social, servies par la collecte puis le traitement de l'information, en vue de l'action sur la société ${ }^{4}$ " L'ennemi intérieur» est l'adversaire dans l'ombre, qu'il faut porter au jour: quelle meilleure expression de l'activité de police, de la surveillance, de l'intelligence. Son emploi par les "professionnels de la sécurité » ne devrait donc pas surprendre. «L'ennemi intérieur » est une figure, au simple sens rhétorique du terme, qui n'indique rien de plus qu'une simple économie de langage.

Mais voilà: trois éléments d'inégale importance viennent aujourd'hui déposer "l'ennemi intérieur» sur la table de travail du sociologue. Le premier est une croissance, dans l'ordre du discours : les professionnels de la sécurité, mais aussi des 
politiques, emploient aujourd'hui ce terme avec une passion hier sans exemple. Cette inflation du vocable signe leur aspiration à tisser un ruban de Möbius où intérieur et extérieur s'enchevêtreraient jusqu'à ne plus former qu'un seul, invitant dans le même mouvement police et armée, sécurité et défense, ennemi et délinquant, à ne faire, chacun dans son ordre, plus qu'un. Le second phénomène, à la fois effet et cause du premier, est l'inflation des dépenses de sécurité, l'indifférenciation des agences de sécurité, l'effacement de la distinction entre prévention et répression. Ces deux phénomènes, Didier Bigo les illustre parfaitement dans l'un de ses écrits ${ }^{5}$. Mais il en évoque aussi un troisième, sous forme d'inquiétude: "peut-on penser le politique autrement qu'à partir de la crise et de l'exception?». En effet, l'emballement contemporain des professionnels et des politiques de la sécurité pour «l'ennemi intérieur » serait le signal d'un changement épistémique dans le champ de la sécurité : mutation des discours (quoi que née de la seule inflation d'un terme ancien, dont on ne sait pas bien la genèse ni le parcours), modification des pratiques (quoi que sensible dans certains domaines seulement du champ, comme la coopération internationale des polices ou la lutte contre la criminalité internationale), revendication de ces changements par l'importation d'une philosophe politique propre, le décisionnisme façon Carl Schmitt.

$C^{\prime}$ est cette superposition de trois phénomènes (vocable qui se multiplie, industrie qui s'y greffe, communauté épistémique qui se proclame) qu'il s'agit ici de discuter. Plus précisément, la présente contribution prend pour objet le domaine de validité de cette sociologie critique de l'ennemi intérieur. Y a-t-il véritablement, attestée par la nouveauté de son vocabulaire et de ses pratiques, emprise du champ de la sécurité par une communauté épistémique, aujourd'hui en voie d'être démasquée? Il faudrait ici partir de la substance, ou d'une définition substantielle, de l'ennemi intérieur, et, autant que possible, la tester; partir, puisque c'est d'elle qu'il s'agit, de la définition donnée par Carl Schmitt.

L'ennemi est chez Schmitt ce qui fonde l'ordre politique. L'ennemi, dans cette perspective, est celui contre lequel l'État, l'institution politique en soi, la seule institution politique qui soit, accède à l'existence et garantit sa pérennité (BdP, pp. 46-47) ${ }^{6}$. Il est celui qui permet à l'État, qui le qualifie comme ennemi et le traite comme tel, de mettre un terme à la situation exceptionnelle et de restaurer les conditions ordinaires de la vie sociale, de restaurer l'ordre public, la «tranquillité, la sécurité et l'ordre $»^{7}(B d P$, p. 46). Schmitt écrit même: «Cette nécessité de pacification dans l'ordre intra-étatique conduit à ce que, dans les situations critiques, l'État n'est unité politique que pour autant qu'il définit de son propre chef 'l'ennemi intérieur' » (BdP, p. 46), et qu'il lui destine tout un ensemble de politiques qui peuvent relever de la proscription, de l'ostracisme, du ban, de la mise hors-la-loi (p. 47). Deux brefs éléments sont à retenir ici. Tout d'abord, l'ennemi ne gagne son statut que par la décision qui le vise : il ne requiert aucune autre propriété singulière que celle d'être ainsi par autrui baptisé. Ensuite, il faut noter l'indifférence aux moyens de traitement de l'ennemi, mais l'importance du critère situationnel : c'est la «situation critique » qui, en appelant le rétablissement de l'ordre, amène à la nécessité de disposer de l'ennemi intérieur. Vouloir une "sociologie critique ", qui ne s'en tienne pas qu'aux mots, de «l'ennemi intérieur » exige donc que l'on étudie ce qui se passe exactement dans ces situations où le bon ordre est troublé. Ces situations, où quelque chose se passe qui ne devrait pas se passer ou qui devrait se passer autrement, sont les situations qui, on le sait, appellent l'intervention de la police ${ }^{8}$. 
A garder les yeux rivés sur les situations en soi exceptionnelles, celles surchargées de technologies de pointe et de dispositions juridiques d'exception (aéroports, terrorisme, trafic de drogue transfrontalier, contrôle des frontières...), on n'est au fond pas surpris de n'y trouver que ce que l'on y cherche: de la coupure et de l'exception, de la clôture et de l'extraordinaire. On y trouve aussi, bien sûr, ces policiers d'élite, ces policiers coupés de l'ordinaire de leur profession et de leur métier, qui se bercent des nouvelles menaces, des risques planétaires, des ennemis intérieurs. On triomphe alors d'un dévoilement, qui découvre démasqués des acteurs repus de technologies imprenables, à l'ombre de dispositifs juridiques faits pour eux, prophétisant à qui veut l'entendre, dans leurs enclaves de maximum security society ${ }^{9}$, les dangers de l'ennemi intérieur. Il $\mathrm{y}$ a deux risques à cette posture de recherche, qui finalement trompent quant à l'ampleur des phénomènes observés. Trop content d'avoir vu une conjonction de discours et de dispositifs pratiques, on conclut vite au changement épistémique, sans voir pourtant que ces dispositifs pratiques ne se convertissent pas à l'identique en activités pratiques. Or, à y regarder vraiment de près, à mesurer par exemple la production pénale de ces agents d'élite, on ne peut qu'être surpris de constater la permanence des répertoires d'action, la pérennité des groupes poursuivis, la maigreur des volumes d'activité, bref : l'écart considérable entre les dispositifs pratiques et les pratiques ${ }^{10}$. Le changement épistémique n'est donc pas tant que l'on croit à relever là où l'on pense le trouver. Ensuite, et surtout, tout indique que le regard ne se porte pas au bon endroit. S'il est un ennemi intérieur, il est partout et toujours où l'ordre se trouble, partout et toujours où l'Etat arrête son statut et décide de son destin. Il faut détourner le regard, le porter loin de ces situations a priori d'exception et le poser là où, dans l'ordre quotidien de la vie sociale, le bon ordre et la tranquillité publique se froissent. Que se passe-t-il alors?

La rue est ce lieu d'épreuve des situations ordinaires. Tout l'enjeu est de comprendre ce qui s'y joue lorsque l'ordre y est menacé ; également de repérer s'il y a aujourd'hui conjonction, inconnue hier, de pratiques, discours et acteurs nouveaux. Le texte que l'on va lire présentera donc successivement des tactiques anciennes de restauration et maintien de l'ordre, puis des tactiques contemporaines, tentera de repérer qui en est l'objet et qui en décide et conclura à l'existence, au sein de notre ordre public ordinaire, de situations de bans, qui en appelleront à Carl Schmitt, mais pour mieux démentir l'usage sociologique de la notion d'ennemi intérieur.

\section{RESTAURER LA TRANQUILLITÉ, LA SÉCURITÉ ET L'ORDRE : LES fiLLES DANS L'ESPACE PUBLIC, fIN XIXE SIÈcLE}

Dans son ouvrage sur la prostitution féminine au XIXe siècle, Alain Corbin évoque ce qu'il appelle «la chasse à la femme ». La chasse à la femme est une tactique policière qui amène les « filles ", comme l'évoque ce passage du livre, à quitter certains quartiers pour en regagner d'autres, se voir alors regroupées avec des collègues d'infortune sur des territoires bien particuliers qui les exposent à des « rafles » policières rendues plus faciles que les risques d'erreur (interpeller une simple passante) sont minimisés :

« Les interdits qui pèsent sur les filles en carte sont si nombreux, la différence entre l'exercice solitaire et licite du racolage et la provocation sur la voie publique est si minime, les quartiers où les isolées ne doivent pas circuler sont si vastes que les malheureuses se trouvent, répétons-le, soumises à l'arbitraire des agents. Le grand nombre des arrestations le prouve à l'envi et c'est cet arbitraire qui pousse les filles 
en carte à 'disparaître'. Afin précisément d'arrêter les disparues et les clandestines, les inspecteurs de la brigade des mœurs, puis les agents de sûreté organisent des rafles, en particulier sur les grands boulevards, les boulevards extérieurs et la sortie des bals les plus fréquentés par les filles (...). Ces 'opérations d'épuration', pour reprendre l'expression de Camescasse ${ }^{11}$, que la préfecture de police ne nie que mollement et qui se déroulent même en plein jour, entraînent de graves bévues et suscitent l'indignation des abolitionnistes ; il faut dire toutefois qu'elles sont le plus souvent organisées à la demande des habitants et, plus précisément, des commerçants de quartier $»^{12}$.

Il y aurait naturellement beaucoup à dire de ce court passage. Notons d'abord la résistance qu'opposent les filles à la catégorisation administrative (sont-elles «en carte »? Ou sont-elles seulement « à numéro » donc en maison close, sur le chemin de leur lieu de travail sans mériter l'attention policière ?). Une première difficulté est donc ainsi constituée par le statut de la fille. Une seconde est liée à son activité (« exercice solitaire et licite » ou bien « provocation »?). Des deux difficultés, et c'est ce qui compte ici, le policier est seul juge. Il lui appartient d'estimer, au moyen de son sens ordinaire des situations $\mathrm{s}^{13}$, le statut et l'activité de la fille et ainsi de déterminer s'il y a matière à vérification d'identité (opération de police administrative), à interpellation (opération de police judiciaire), ou à expulsion hors du «quartier où les isolées ne doivent pas circuler $"$.

C'est ce dernier type d'action policière qui est l'objet de la présente contribution, puisque c'est par lui que semble se gérer, au quotidien, les situations quotidiennes critiques. Le policier peut procéder à une mesure de déplacement de la fille (« rafle » ou "opérations d'épuration»), ou bien d'interdiction du territoire où elle se trouve au moment de la rencontre. Il s'agit d'une opération dont le flou de la définition juridique fait écho à l'indétermination du statut et de l'activité de la personne visée : elle est à la fois une mesure de police administrative, en ce qu'elle s'effectue dans le cadre du pouvoir réglementaire concédé par la loi aux agents de police ${ }^{14}$ et qu'elle échappe, de ce fait, au contrôle du juge; elle vise également à sanctionner un délit (de "provocation»), comme tel défini dans le Code pénal et appelant mesure de police judiciaire.

\section{Les territoires policiers}

La «chasse à la femme» illustre l'ancienneté d'une relation spécifique entre des pratiques policières au fondement juridique mal défini et des pratiques qui consistent à exercer à l'égard de certaines populations des stratégies de déplacements et d'assignations. La constitution des «territoires policiers" vise donc des gens particuliers à des fins de connaissance (savoir où ils sont) et de contrôle (maitriser ce qu'ils font). Qui sont ces gens? Ceux qui se trouvent, pour des raisons extrêmement diverses, objets de l'intervention plus fréquente, de l'attention privilégiée, de la part de la police. Ces raisons diverses ont toutes à voir avec le fait que, aux yeux là encore de la police, ils peuvent représenter un trouble à la tranquillité publique, ou bien avoir commis un délit. En ce sens, nous pouvons, à la suite d'autres auteurs, les définir sous l'appellation de "clientèle policière $»^{15}$. Mais nous butons à cet endroit sur un problème, qui ne peut être pour l'instant résolu. Problème qui tient à l'action des policiers, puisqu'il s'agit de déterminer les compétences qui fondent leurs pratiques de sélection : nous verrons plus loin ce que la loi dit là-dessus. Problème qui tient ensuite à 
l'analyse par le sociologue, puisqu'il s'agit d'interroger ces personnes dont la propriété discriminante est qu'elles se trouvent en relations fréquentes avec la police.

\section{A la recherche des populations pertinentes : conditions du repérage sociologique}

Ne considérons pour l'heure que la difficulté méthodologique. Les quelques extraits d'entretiens que l'on va présenter sont le produit d'une enquête sur l'usage de la police en France ${ }^{16}$. L'un des choix de cette enquête avait été d'interroger des personnes pouvant témoigner d'interactions personnelles avec la police, témoignages appelés à constituer un matériel discursif permettant de comprendre un peu mieux les logiques d'interactions policières. La stratégie de recherche ne consistait alors pas à savoir combien, d'une population générale, ont déjà eu recours à la police, se sont déjà fait contrôler sur la voie publique, ou interpeller ; les réponses à ce type de questions, qui visent la fréquence des interactions policières, sont établies depuis longtemps. Il importait au cours de cette recherche de déterminer ce qui se passe lors des interactions policières brutales, afin de dessiner la morphologie de ces interactions, d'en comprendre les raisons et d'en définir les logiques.

Une telle problématique imposait une sélection de nos interlocuteurs, déterminés en fonction de leur forte probabilité à avoir connu de telles interactions avec la police. $\mathrm{C}^{\prime}$ est ce qui nous a conduit à interroger des personnes sorties de prison, personnes dont la propriété la mieux partagée est d'avoir été interpellées, une fois au moins au cours de leur existence, par des services de police ou de gendarmerie. Une telle sélection des personnes interrogées répondait d'abord à la nécessité dictée par l'économie des moyens. Mais cette nécessité a une vertu. Elle nous amène, précisément, auprès de ceux qui ont appelé l'attention de la police, et la provoquent peut-être encore, en diverses occasions. S'il fallait résumer d'un trait les critères de constitution de notre corpus, nous dirions que ce groupe, qui n'a d'autre principe de constitution que de rassembler ceux que la police rencontre, peut nous amener à comprendre, par les entretiens individuels, quelques unes des logiques constitutives à l'interpellation et, au-delà, aux relations entre les policiers et leur « clientèle ».

Le principe de sélection, qui répond à un souci général d'économie de la recherche, engendre deux instruments de sélection. Le premier d'entre eux est préalable à l'intervention du chercheur : il s'agit de la décision d'incarcération, qui dans un schéma idéal naît du délit commis et se conclut par la décision du juge pénal. Le second instrument de sélection a été en revanche guidé par les circonstances. A l'époque de la recherche (1994-98), l'agitation sociale des surveillants de prison rendait impossible les entretiens menés dans les centres de détention. Nous avons donc interrogé non pas des détenus, mais des sortants de prison, bénéficiaires d'aides pourvues par un service de la ville de Paris et du ministère de la Justice, le S.R.A.I.O.S.P., service d'assistance aux sortants de prison marginalisés ou sans ressources. Sa fonction est d'accueillir les anciens détenus en situation difficile et de les aider à trouver le plus souvent un logement et dans le meilleur des cas, un emploi. Le service fait également office de centre de réception du courrier des personnes qui s'y adressent, permet à ces personnes d'y goûter quelque repos (dans la salle d'attente, où un poste de télévision diffuse des vidéos de films animaliers). C'est auprès de cette population de sortants de prison, dans ce centre, que nous avons conduit nos entretiens. 
La population accueillie au S.R.A.I.O.S.P. présente deux caractéristiques importantes. D'une part, elle rassemble des personnes, par définition, en marge de la société: seulement un Français sur mille a subi une peine de prison ferme en $1994^{17}$. Leur exacte situation pénale renforce cette marginalité puisque, en $1994,84 \%$ d'entre eux sont récidivistes, $14 \%$ seulement "délinquants primaires $»^{18}$. La durée de leur incarcération souligne une évidente rareté des lourdes peines : 6,5\% seulement ont subi une peine supérieure à 3 ans. Les deux tiers des bénéficiaires de l'aide du S.R.A.I.O.S.P. ont subi des peines de 1 à 12 mois $^{19}$. Ce ne sont pas des délinquants criminels, mais des personnes souvent familières de petits délits; vol à la roulotte, vol à la tire, vol à l'étalage, consommation, détention ou trafic de stupéfiants, recel, faux en écriture... Il faut rappeler à titre de comparaison qu'au premier janvier 1994, 12\% des détenus français étaient incarcérés pour une durée supérieure à trois ans et $31 \%$ pour une durée comprise entre un mois et un an. Nos enquêtés sont donc principalement caractérisés par une délinquance de voie publique, fréquente et à maigres profits, propice à provoquer un repérage aisé par la police de voie publique : beaucoup ont été présentés au juge dans le cadre d'une procédure de délit flagrant. Notre population, de par ses caractéristiques pénales, se distingue donc en ceci qu'elle a toutes les chances d'avoir une relation fréquente avec les policiers.

Cette population se distingue également sur le plan social. Ces personnes affrontent des situations d'exclusion ou de marginalisation sur les marchés du travail et du logement, quelques uns requièrent également le soutien de l'association dans le domaine de l'accès aux soins médicaux. L'identification de notre population n'est pas simple, en outre, sur le plan de la nationalité. En effet, si la plupart des requérants sont Français ${ }^{20}$, beaucoup sont d'origine étrangère ou nés de parents étrangers et ont perdu leurs papiers ou quelques éléments de leur état-civil. La situation en termes de travail est dramatique: ceux qui bénéficiaient d'un emploi avant leur incarcération, peu nombreux (6 des 32 que nous avons interrogés), ne le retrouvent pas à leur sortie de prison (sauf une seule exception). La marginalité de cette population est plus forte encore que celle des autres détenus, puisque, en mars 1993, 57\% des condamnés étaient actifs au moment de l'interpellation. Enfin, les trois quarts des requérants, en 1994, ont demandé un logement à l'association compétente. Lorsque celle-ci parvient à offrir une solution à ce problème (le plus souvent grâce à différents foyers), les perspectives restent sombres pour les bénéficiaires. A la sortie du séjour pris en charge par Le Verlan, un quart des bénéficiaires ne sait où aller, $15 \%$ se trouvent dans une situation inconnue et un tiers multiplie les séjours dans divers foyers.

Cette marginalité sociale se marque, au sens propre du terme, par des stigmates particuliers, repérables chez bon nombre de personnes interrogées: dentition très irrégulière, traces de blessures au couteau ou d'automutilation aux poignets ou aux avant-bras. C'est à dessein que nous employons ici le terme de "stigmate", au sens retenu par Goffman : porté par la personne qui ne peut s'en défaire, il est un «signe fort » du lien tissé avec ceux qui ont en charge de surveiller, au sein de l'espace public, les personnes qui s'y meuvent ${ }^{21}$. Tous ces éléments, en un mot, permettent de croire que nous disposons là d'une population à même de témoigner des mécanismes de repérage et de traitement, par la police, de ceux qui troublent. C'est la raison pour laquelle, au lieu des attributs classiques par lesquels la sociologie qualifie les personnes (sexe, âge, profession, diplôme), seront ici mentionnés les attributs secondaires, ceux de façade, par lesquels ces personnes se donnent à voir dans l'espace public (couleur de peau, "signes particuliers») et se prêtent à l'identification auprès des policiers 
(caractéristiques pénales). Afin de comprendre au mieux comment le lien se tisse à partir des signes pertinents, nous reprenons ici les catégories que la police utilise sur les avis de recherche (les «types » et les « signes particuliers ») 22 .

Si les conditions de sélection de la population enquêtée ont ainsi été sommairement exposées, il restera à dire un mot de la parole reçue, de la validité du matériel d'entretien ainsi recueilli. Il y a bien sûr quelque illusion à vouloir régler cette question avant d'avoir pris connaissance de ce matériel et de ce que ces gens, interrogés, disent vraiment. Posant le problème de la manière la plus simple, nous soulignerons simplement la force du silence qui entoure l'objet initial de notre recherche, l'usage de la force par la police. Ce silence tient à la nature de ces événements : rapides, confus, indistincts, ils entraînent une prise de parole nécessairement polémique. Moins encore qu'ailleurs, ici, le témoignage ne fait preuve. Le silence est aussi le produit de l'inégale attestation sociale et juridique de crédibilité des paroles entendues, celles, souvent les seules en concurrence, du policier et de la victime putative ${ }^{23}$. Le silence est enfin ce qui enveloppe le traitement des événements, leurs conséquences: par anticipation des chances de succès autant que par les coûts d'une telle mobilisation, les victimes déclarées s'en remettent peu à la justice pénale ou administrative, si bien que les archives des Cours ou des corps disciplinaires se révèlent d'un maigre secours lorsqu'il s'agit de valider de tels entretiens. Mais, à l'inverse, la validation systématique du témoignage individuel par renversement de l'administration de la preuve induirait tout autant en erreur: si le témoignage oral ne peut que trop rarement être validé par d'autres moyens (le témoignage oculaire ou la procédure judiciaire), il serait aventureux de lui prêter foi a priori.

Comment faire? Voici des témoignages, sources de renseignements sur les relations de populations particulières avec la police, dont l'indice de crédibilité reste invérifiable, mais dont la valeur d'usage est définie par la problématique même qui a invité à ces entretiens : seuls ces témoignages renseignent sur ce que l'on veut savoir, leur prix est donc inestimable. Que faut-il choisir, et c'est là le nœud épistémologique d'un tel matériel, d'une valeur d'usage indexée à la rareté du bien dont on dispose, valeur d'usage élevée, et d'une valeur intrinsèque ruinée par l'impossible validation de chaque unité de ce bien? On nous permettra ici de ne pas trancher le débat épistémologique, ou plutôt d'en déplacer les termes, de les rapporter exactement à l'enjeu de connaissance qui est le nôtre ici : connaître les interactions de la police et de groupes particuliers, comprendre la formation et l'institution de ces relations, et en apprécier la nature politique. On posera d'emblée une entière indétermination de la vérité du fait rapporté : on ne peut savoir s'il est vrai. Et pour le dire de manière tranchée, on supposera d'entrée qu'il ne peut que renseigner sur les représentations propres à celui qui le rapporte, qu'il ne dit vrai, au fond, que de l'imaginaire individuel. Mais la force sociale de cet imaginaire est telle qu'elle n'est pas sans effet sur le comportement individuel, autrement dit la faculté de se trouver pris dans des schèmes de perception spécifiques à l'égard de la police (on verra lesquels précisément) n'est pas sans effet sur le comportement effectif en cas de rencontre avec la police. Car de la représentation au comportement, l'ensemble des anticipations et calculs sur la manière dont les choses vont se dérouler se posent en médiation entre les événements imaginés et les événements vécus, de sorte que ces derniers entretiennent avec les premiers une relation de probabilité. Ce ne sont donc pas des faits que nos entretiens livrent ici, mais quelques unes des conditions de probabilité d'occurrence de certains faits spécifiques. L'émotion, qui est l'élément de médiation entre la représentation et le comportement, 
est une des données fréquentes de ces entretiens lorsqu'il est question de police. Mais elle est aussi l'un des outils de travail des policiers, en ce sens qu'elle permet le repérage par ces derniers des populations vers lesquelles elle se tourne. Le vain dialogue entre le fait imaginé et le fait vécu, entre la valeur intrinsèque et la valeur d'usage de l'entretien, se clôt ainsi au moment de l'exercice de leurs compétences par les policiers qui, fondées sur des techniques de repérage des déviances, ont tôt fait de convertir l'émotion de ceux qu'ils croisent en ressource objective de leur action, selon des mécanismes qu'il faut à présent examiner.

Perceptions des pratiques policières : les tactiques de territorialisation

Ce bref témoignage recueilli auprès d'une prostituée, consommatrice d'héroïne et de crack, rappelle sans difficulté les pratiques policières du temps du préfet Camescasse, celles d'il y a un siècle :

«- Alors, justement, est-ce que vous avez souvent des contacts avec la police?

- Oh là. Tous les jours.

- Tous les jours?

- Tous les jours pour une simple question que je traîne au quartier de Max Dormoy où se vend le crack et que tous les jours ils nous font des descentes et ils nous tabassent la gueule avec leurs matraques $»$.

Femme, env. 30 ans, type européen,

14-15 condamnations pénales (1ère en 1985)

La fréquence des rencontres avec la police est ici immédiatement liée à la fréquentation d'une aire urbaine précise, le quartier de Marx Dormoy, dans le XVIIIe arrondissement de Paris. Cette aire est celle où se rassemblent, en l'occurrence, des consommateurs de crack. Sur ce territoire, la fréquence des interventions policières est telle qu'un lien tout à fait privilégié s'établit entre les policiers et la «clientèle ", que singularisent des pratiques policières comme l'usage manifestement excessif de la force qu'on ne retrouve pas ailleurs, sur d'autres territoires. Le deuxième témoignage rend compte, quant à lui, de l'effort des policiers visant à expulser un toxicomane hors d'un certain territoire. Le retrait hors de la voie publique et l'atteinte au corps, visant l'humiliation, avaient selon la personne interrogée pour objet d'élever considérablement le coût de son maintien dans ce quartier, jusqu'à y rendre sa présence intenable.

« - Et lorsque le type vous attendait à la sortie de la pharmacie?...

- Ouais, ils étaient deux. Ils étaient deux. Toujours les deux mêmes.

- C'étaient deux hommes?

- Deux hommes, ouais. Un petit brun et l'autre grand, blond. Et puis, je l'avais déjà vu plusieurs fois, quoi, mais... quatre jours de suite... mais je me sentais vraiment persécuté, je me disais, mais... Soit quelqu'un a dit des choses sur moi, je sais pas quoi, et... ils pensaient que j'étais un dealer ou je sais pas quoi. Moi, je m'achetais ma petite seringue, pour être hygiénique, je m'envoyais des cachets, c'était des Temgésic, hein, je sais pas si vous connaissez et voilà, je m'injectais ça. Et, quatre jours de suite, dans le même escalier... en plus c'était la cage d'escalier de mon... médecin... traitant, juste à côté de chez moi. Et ils me désapaient comme ça dans les escaliers à poil, tout à poil et puis ils me fouillaient partout, ils étaient contents et... 'Allez, casse-toi, maintenant...' Quatre jours de suite, je trouvais ça un peu baisé. Si ils avaient vraiment quelque chose contre moi, ils m'embarquaient, ils m'amenaient vraiment au commissariat et puis voilà. Là, c'était quand même assez illégal de, de...

- Et vous protestiez?

- Comment?

- Vous protestiez, vous?... 
- Ben... La première fois, j'ai pas protesté, hein, mais la deuxième fois, les... les... les trois autres fois, j'ai dit: 'Bon, soit vous m'amenez au commissariat, vous avez quelque chose contre moi, soit...'. 'Ferme ta gueule', comme ça, 'Tu te déshabilles, point à la ligne, je suis sûr que t'as de la came, allez vas-y, machin', enfin voilà. Je me désapais, j'étais congelé, il me fait : 'C'est pas grave, ça te fais du bien, machin'. Alors, je crois que... Je sais pas si ils jouent avec moi ou quoi, $\mathrm{j}$ 'en sais rien, mais bon. (...)

- Et lorsqu'ils vous quittaient, là...

- Oh, bah c'était du genre, 'Ouais, on veut plus te revoir, hein'. Alors que c'était mon quartier. 'On veut plus te revoir, hein, ici, machin'. Voilà, c'était ça.

Homme, 27 ans, type européen, plusieurs condamnations pénales, extrêmement nerveux.

On peut par cet entretien un peu mieux comprendre l'établissement des territoires policiers. Toxicomane, habitué à s'injecter des substituts de morphine vendus en pharmacie, il se rendait chaque jour à la pharmacie de son quartier pour acheter une seringue. Ce faisant, il attirait la suspicion des officiers de police judiciaire en service sur cette circonscription ${ }^{24}$, sans pour autant être pour eux d'une grande utilité. Le travail de police judiciaire, en effet, a pour objet de sanctionner les délits, et la consommation de stupéfiants en est un. Mais les policiers, qui ne peuvent tout poursuivre, rationalisent leurs activités, selon un principe d'utilité, fondé sur les avantages comparés de la poursuite de tel ou tel délit: le grand criminel fait le grand policier, et l'appréciation des performances professionnelles par la hiérarchie interne et par le procureur est indexée à la valeur des crimes résolus et des délinquants interpellés ${ }^{25}$. C'est précisément l'utilitarisme de l'action, fondé sur une évaluation des rendements propres à chaque interpellation, qui assigne notre interviewé à l'entredeux juridique de l'action policière et conduit à le voir objet d'une stratégie de territorialisation. La personne interrogée rend bien compte de l'incertitude des fondements juridiques de l'action policière, en insistant sur le fait que le délit qu'elle commet est sans doute de trop petite «taille » pour motiver une procédure judiciaire : "Si ils avaient vraiment quelque chose contre moi, ils m'embarquaient, ils m'amenaient vraiment au commissariat et puis voilà. Là, c'était quand même assez illégal de, de... $\aleph^{26}$. Il manifeste également la plus grande indignation devant l'acharnement des policiers, car cette stratégie de déplacement est rendue d'autant plus insupportable que, précisément, il est là chez lui. Mais aux yeux des policiers, son statut de consommateur de stupéfiants surdétermine son ancrage géographique. Il est le critère qui détermine l'appartenance à une clientèle et l'enjoint à quitter le territoire familier pour gagner un territoire policier.

On le voit à l'aune de ces deux exemples: les pratiques de déplacements et de regroupement dans l'espace urbain de populations données coïncident avec des mesures policières situées quelque part entre police administrative et police judiciaire. Ces pratiques s'appliquent à des populations que l'activité (prostitution ou consommation de drogues) semble assigner à la compétence exclusive de la police. Ces deux dimensions devront être expliquées, car elles éclairent le sens des stratégies topographiques employées par la police. Avant cela, nous pouvons d'ores et déjà, sur la base des entretiens menés avec les sortants de prison, décrire les logiques propres à ces stratégies, en quoi elles consistent. 


\section{Déplacer et regrouper : les stratégies policières et leurs effets micro-sociologiques}

\section{Le territoire efficace}

"L'espace occupe dans le droit une place importante et souvent méconnue », lit-on dans un manuel de théorie juridique ${ }^{27}$. Le principe de territorialité de la force publique, le ressort territorial sur lequel peut s'exercer une compétence de police, est un élément formel essentiel de la sauvegarde des libertés publiques. Mais, « à cette préoccupation d'ordre quasi-philosophique(sic!),s'ajoute une préoccupation plus pragmatique, celle de tendre vers une gestion plus rationnelle de la police ", ajoute un manuel juridique destiné aux policiers ${ }^{28}$. Cette "gestion plus rationnelle" amène à ces stratégies complexes de dé-territorialisation et reterritorialisation, dont il faut à présent rendre compte dans le détail.

Le premier objet de l'action policière est la rationalisation de l'espace, qui consiste à rendre le milieu environnant compréhensible, opératoire, puis exploitable. Il s'agit tout d'abord de circonscrire la délinquance sur des aires urbaines délimitées, afin d'accroître la visibilité des délinquants, par leur inscription dans un endroit techniquement praticable (propre à l'observation de loin, avec des couloirs d'accès rapides et discrets) et socialement peu coûteux (en marge des habitations où les populations locales disposent de ressources suffisantes pour mobiliser les pouvoirs publics sur la question de la sécurité). La place Stalingrad, avant sa rénovation, les abords du boulevard périphérique, comme la Porte de la Chapelle, sont ces endroits qui ont été très fréquemment cités dans les entretiens. Cette territorialisation est doublement efficace. La spatialisation occupe d'abord une fonction très nette de classement des populations, où le policier retrouve facilement sa clientèle et les marginaux leurs congénères. Ensuite, le territoire facilite la formation d'un marché, en assurant aux vendeurs et aux consommateurs un espace propice aux échanges, qui tient à l'écart ceux qui ne vendent ni n'achètent. Ce marché permet à la police la visibilité des entrants et des sortants, ainsi que la limitation de l'éparpillement des activités illicites liées à la drogue ${ }^{29}$. Ainsi, les manœuvres de territorialisation ont également pour conséquence la réduction des risques que la police associe à la libre circulation des biens illicites et de ceux qui en font commerce. La détermination de la part des délinquants de leur inscription géographique et de leur mobilité, analysées en soi comme source de nuisances :

«Moi, ce qui m'én..., ce qui m'énerve un petit peu, moi, c'est que... le dealer, il est reconnu. Nous, on sait que c'est lui qui deale, nous on sait que c'est lui qui vend, mais c'est pas lui qu'on va interpeller. Des fois, ils vont laisser... Enfin, c'est comme ça que j'ai analysé, hein, je dis pas que c'est la vérité vraie, mais on va laisser un dealer vendre pendant un certain temps là, parce qu'il faut qu'il y ait un dealer à un endroit qui puisse vendre pour le toxicomane, parce que si le toxicomane quand même ne trouve pas sa drogue, il va faire encore plus de délits parce que, si il a pas d'argent, il trouve pas son produit ».

Homme, env. 30 ans, type nord-africain, a séjourné aux maisons d'arrêt de FleuryMérogis et de Fresnes, trois doigts sectionnés main gauche, dents manquantes.

L'espace urbain s'organise sous l'effet de l'action de la police en autant de pôles attractifs et répulsifs pour les sortants de prison. Et à l'économie de l'action policière répond sur ces territoires la structuration de marchés illicites (drogues ou prostitution dans le cas de deux femmes de la place Stalingrad et de Marx Dormoy). Là réside la 
première source de difficultés soulevées par ces stratégies de territorialisations. Car la maximisation des rendements se retrouve commune aux policiers et aux délinquants : les premiers rationalisent un espace urbain indistinct, en le découpant en zones identifiables; les seconds voient le marché des biens illicites (stupéfiants et recels) coïncider avec une topographie bien déterminée.

On comprend donc comment certains modes de gestion des désordres de voie publique trouvent leur solution par ces stratégies territoriales, stratégies de répartition des publics concernés dans la ville. Qui sont, toutefois, ces publics concernés?

\section{L'espace social et l'anomie urbaine}

Quelles sont les personnes qui se trouvent visées par ces stratégies de territorialisation? Comment s'opère, puisqu'il s'agit bien de cela, leur sélection ? Il faut mentionner ici un trait particulier à la population interrogée: engagés dans une carrière pénale souvent bien dense, ces personnes, exclues des marchés du travail et du logement, également du marché matrimonial, manquent d'une ressource essentielle: l'espace privé, ou plus précisément de la possibilité de disposer d'un «territoire du moi $»^{30}$, lequel est la garantie de ressources nécessaires à affronter une vie par ailleurs difficile (retrait, intimité, repos, etc.). Aussi, la propriété commune de ces personnes est la très grande difficulté à disposer d'un espace privé. Par leur inactivité, par la précarité de leur logement ou l'absence de logement, mais également par le fait que leurs ressources et leurs consommations (notamment de stupéfiants) leur sont seulement accessibles sur la voie publique, ces personnes passent le plus clair de leur temps dans la rue. Ils partagent cette propriété avec, bien sûr, les seuls fonctionnaires qui, avec ceux de la voirie, ont précisément pour tâche d'exercer sur la voie publique : les policiers. Ces derniers ont alors à gérer une population bien particulière, vivant dans un régime d'anomie, si l'on comprend ce terme dans l'acception que lui donnait Durkheim: état d'isolement, mais aussi d'indiscipline et de dérèglement ${ }^{31}$. Par les mécanismes de maximisation commune des rendements, la territorialisation contribue à une stratégie de discipline de ces individus, par leur résidence sur des territoires déterminés. Elle opère par la régulation de leurs itinéraires urbains une régulation sommaire de leurs activités sociales.

Cet effet se double d'un second, qui touche comme on l'a indiqué plus haut, à la perception de soi des individus. La territorialisation produit un double effet de manifestation et de confirmation du stigmate. La plupart des entretiens évoquent la fréquence de ces techniques de gestion de l'apparence de la part des policiers et, en retour, des marginaux. Pour ces derniers en effet, il importe plus que tout de ne pas provoquer d'alarme et ne pas provoquer la curiosité des policiers : il faut, comme le dit Goffman, que l'apparence "cadre » avec les représentations policières de la normalité comportementale ${ }^{32}$. Or, l'apparence est perçue chez les personnes interrogées comme le stigmate de l'appartenance au monde des délinquants : soit que la consommation de drogue ait altéré les cheveux, la peau, le regard, soit que la violence de ce monde se repère en autant de cicatrices et hématomes, les policiers jouent dans ces rencontres le rôle de révélateurs (et confirmateurs) des stigmates. Arrêter le cours de la marche d'un individu sur la voie publique pour lui demander ses papiers, puis lui indiquer qu'il n'a rien à faire là, ou le retenir dans le véhicule de service voire au poste de police pour vérifications approfondies (art. 78-3 Code de procédure pénale)... toutes ces pratiques qui happent ne serait-ce qu'un instant l'individu hors de la normalité de l'aller et venir 
ordinaire le rappellent à son statut de marginal, de sortant de prison et, de ce fait, de membre de la clientèle policière ${ }^{33}$. Le territoire vers lequel la personne se trouve ensuite conduite joue aux yeux des personnes interrogées la preuve manifeste de la marginalité sociale. Il y a donc, pour conclure sur ce point, rencontre du stigmate, de l'espace social et de l'espace géographique : à la marginalisation territoriale répond la marginalisation sociale, et inversement. Les pratiques de territorialisation aboutissent à une identification du statut de la personne non plus fondée sur ses attributs ordinaires d'appartenance (la nationalité française, par exemple), mais sur ce que l'on appelle dans la sociologie classique les $"$ attributs secondaires $»^{34}$. Le travail quotidien de la police amène l'ordre des apparences à surdéterminer l'ordre des identités sociales ou nationales. La territorialisation, en fin de course, manifeste et confirme l'effectivité de ce travail, en groupant sur un même lieu ceux qui se ressemblent. Cet extrait d'entretien le montre parfaitement, où l'on voit le lien ténu entre la rupture des apparences lue sur le visage de la personne, l'intervention policière qui en découle et enfin l'invitation à la déterritorialisation formulée par les policiers.

«- (...) Parce que le type, il vous attrape dans la rue, contrôle vos papiers, ils vous mettent une tête et après ils vous relâchent, vous n'existez pas. Surtout avec la gueule que j'ai.

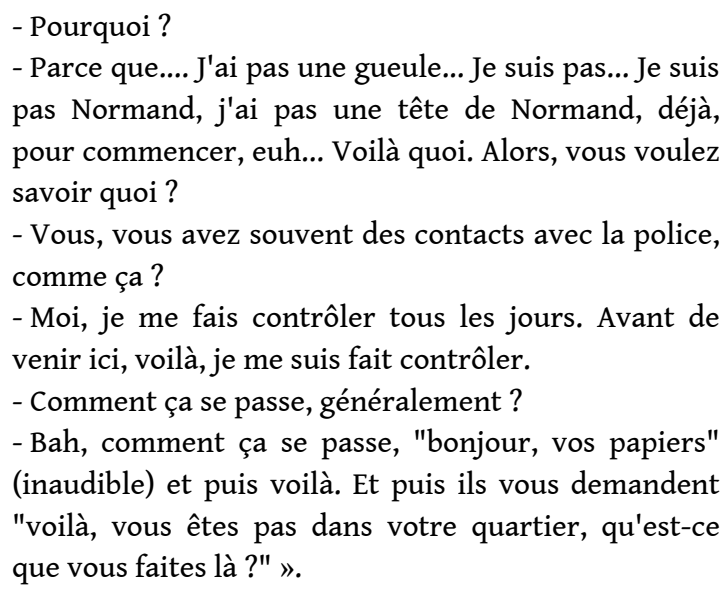

Homme, 36 ans, Français, type nord-africain, trois peines de prison fermes exécutées,

lèvre fendue en plusieurs endroits, arête nasale déformée.

On remarquera ici, sans insister sur ce point qui nous emmènerait trop loin, que la territorialisation confirme le statut social par la surdétermination des apparences, mais qu'il joue aussi, évidemment, un rôle très important en ce qui concerne la légitimité à se trouver dans tel espace national : les apparences, ce sont bien entendu en premier lieu la couleur de la peau. Il n'est pas de meilleur exemple, en la matière, que celui des stratégies de mobilité dans l'espace urbain que celles des étrangers en situation irrégulière. On voit dans cet extrait d'entretien réalisé par Johanna Siméant, qui montre qu'échapper à la territorialisation policière impose à la fois une tactique de surdétermination de la normalité et de mobilité spécifique :

« Les gens descendaient à Porte de Bagnolet et ils allaient à pieds jusqu'au Sentier pour ne pas se faire contrôler dans le métro, ils se coupaient la moustache, ils allaient se faire faire les cheveux clairs chez le coiffeur. Pour ne pas attirer l'attention ».

Entretien réalisé par J. Siméant le 19 mars 1994 avec un leader de la grève de la faim de 1980, ancien membre de Dev Yol ${ }^{35}$.

Mais ce serait accorder trop peu de place aux dimensions psychosociales de ces rencontres avec les policiers que de ne mentionner que ces aspects du comportement 
des marginaux. Le rapport à la police est, en effet, caractérisé par une émotion intense. Celle-ci est l'effet de la proximité avec la police sur la voie publique, mais elle finit par en devenir, par son intensité propre ; sa cause.

\section{Déterminations micro-sociologiques}

La fréquence des rencontres avec les policiers, la crainte (d'interpellation ou de violences) qu'elles inspirent amènent bien évidemment les marginaux à se voir " pris » dans des territoires. Ce régime de capture permanente dans lequel ils disent vivre a des conséquences importantes sur leurs comportements sur la voie publique, qui comme par miracle se trouvent identifiés par les policiers comme motivation suffisante en vue d'une intervention. La menace de se voir rappelé à son statut et à son stigmate se trouve vécue à chaque rencontre avec les policiers. Les personnes que j'ai interrogées se perçoivent donc dans un rapport de voisinage permanents avec les policiers : peu à peu, l'environnement tout entier devient suspect et les marginaux vivent une sorte d'omniprésence de la police, qui se double d'une omniscience de ses agents. Présents partout, les policiers savent tout, car ils voient, ils suivent, ils traquent. C'est ce qui ressort de ces deux extraits. Le premier témoigne de l'omniscience imputée aux policiers, le deuxième rend compte du fait que les policiers suivaient l'auteur d'un délit qu'ils n'avaient plus qu'à «cueillir», sans effort, certains du délit qu'il allait commettre.

«- Si vous... si vous marchez dans la rue, là... Par exemple, en sortant, là, vous marchez dans la rue. Vous croisez des policiers. Vous sentez quelque chose particulier? Vous avez un sentiment particulier? En les voyant, en les croisant...

- Si. Ah, ouais si. Moi je sais qu'ils sont vicieux.

- Ah bon?

- La vérité. Je les vois vicieux, moi, je les... je les vois vicieux, moi, je les vois vicieux, moi, la police, je... je les vois vicieux. Oui, ils sont vicieux, moi la police, alors euh... C'est pas grave, chais pas.

- Pourquoi ils sont vicieux

- Enfin, ils sont... ils savent déjà la chose... ils savent

déjà calculer la chose, eux.

- Qu'est-ce qu'ils calculent?

- Oh, là ! Calculer... (fin de la cassette)»

Homme, 32 ans, type nord africain, estime la durée totale de ses séjours en prison à 5 ans, démarche incertaine, visage marqué de cicatrices

«- (...) Parce que ça faisait un moment qu'ils me suivaient, quoi. Et que ils m'ont vu rentrer dans... Ils ont pas compris par où je suis sorti, par où je suis rentré. Et quand je suis sorti, j'avais des livres avec moi, que j'avais trouvés, à côté d'un palier... que j'avais pris. Et puis... et voilà, quoi.

- Qu'est-ce qu'ils vous ont dit, là, au moment où ils vous ont trouvé ? Qu'est-ce qui s'est passé ?

- Rien. J'ai eu des coups, seulement (silence). (...)

- Et vous, vous saviez qu'ils vous suivaient?

- Non. Non.

- Pourquoi ils vous suivaient, comme ça?

- Je ne sais pas pourquoi. Ça leur arrive de suivre des gens. Chercher des situations, quand c'est le cas ».

Homme, 34 ans, type européen, trois condamnations pour vols

On ne peut donc comprendre l'intensité sociale spécifique du contrôle d'identité que sous cette perspective. Le contrôle convertit ces représentations en réalité attestée 
dans l'occurrence de l'interaction : comme le soulignait l'un des entretiens cités, c'est après avoir procédé au contrôle de l'identité de notre interlocuteur que les policiers lui ont dit « ce n'est pas ton quartier, ici ». Le moment du contrôle est celui où l'individu contrôlé peut se ressentir comme étant «du dehors » (de la civilité ordinaire dans des espaces anonymes) ou du dedans (de ces territoires policiers - qui rappellent en l'occurrence la réclusion carcérale). Le contrôle d'identité est l'un de ces rares moments de la vie sociale où les représentations, aussi déformées qu'elles soient, rencontrent leur démonstration empirique immédiate. Même si la perception de l'omniprésence policière peut être entendue comme une sorte de paranoïa structurelle :

«- Ah non, mais à chaque fois que... Surtout quand vous avez... Vous avez fait un séjour en... en prison, dès que vous sortez, que vous voyez une patrouille de flics, dans le métro, généralement, vous pouvez être sûr, dans sa tête on se dit qu'on va se faire arrêter. On devient parano, quoi. C'est vrai qu'on devient parano.

- Même si on n'a rien?

- Ah même si on n'a rien, ah ouais. On a beau avoir ses papiers, rien sur soi, machin, on a toujours un truc qui nous travaille, on se dit, on va se faire arrêter. Et trois fois sur quatre, on se fait arrêter. On se fait un contrôle de... Et c'est ça le problème, on se fait arrêter et c'est quoi? C'est donner ses papiers, fouilles corporelles et ça, on en a marre. Je veux dire, bon, on l'a déjà assez vécu et surtout qu'on n'a rien fait, quoi, c'est un truc qui nous prend la tête, enfin moi, ça me prend la tête, c'est... Se faire fouiller comme des... Droit au mur, machin, écartez les jambes, ça va, quoi, on donne. Surtout en prison, quoi, je veux dire c'est chiant.... Surtout en prison, quoi. On se fout à poil, on se met accroupi, faut tousser, c'est assez... C'est assez gênant, quoi. C'est vrai, les gens dehors, ils connaissent pas".

Homme, 30 ans, type européen, deux condamnations dont une pour vols à main armée

On comprend ainsi toute l'intensité de l'anticipation d'une rencontre avec les policiers au moment du contrôle d'identité. Ces sentiments de traque et d'enfermement à ciel ouvert amènent à des comportements bien précis lors des rencontres avec les policiers. Ce nouvel extrait d'entretien l'illustre parfaitement :

«- Lorsque vous aviez été arrêté, le 17 octobre, ça s'était passé comment?

- Jamais. Ce 17, il me vient à l'esprit à chaque fois. J'ai jamais été arrêté, vous savez.

C'était le 23 et le 26 . Le 23, ça s'est passé... Je sortais d'un petit restaurant que j'aime bien et il y avait des fouilles, tout le monde y passait, tout le monde y avait droit. Je voulais revenir au restaurant et après je me suis dit 'Pourquoi se cacher ?'. Et alors j'ai continué sur le même trottoir comme tout le monde. On m'arrête. On m'arrête, c'était des agents en uniforme. Papiers. Comme tout le monde. Alors, j'ai donné mes papiers. Comme tout le monde. Après, on me dit: 'Vous n'avez pas une autre pièce officielle ?' J'ai dit 'C'est tout ce que j'ai'. Alors, il demandait au téléphone, à la centrale, certainement, après on me dit 'Vous montez'. Je monte dans leur camionnette et c'est là qu'on m'a emmené au poste et qu'on m'a confisqué mon press-book ».

Homme, 37 ans, type nord africain, au moins deux incarcérations

Toute l'intensité de ce témoignage se cristallise sur le moment extrêmement précis de la césure, lorsque l'individu contrôlé est happé hors de la voie publique pour être 
emmené en rétention aux fins de vérification approfondie. D'abord, cette intensité est anticipée. Il y a dans la volonté de notre interlocuteur à se porter à la rencontre des policiers qui contrôlent une revendication d'apparence normale : il conteste par là que l'espace urbain puisse être autre chose qu'un espace public où s'éprouvent dans la liberté d'aller et venir les droits à l'universalité et à l'indifférence. L'anticipation du moment du contrôle, peut-on aisément imaginer, est d'une telle intensité qu'elle peut se manifester par des gestes tout à fait peu perceptibles à l'œil non policier, mais qui revêtent une signification particulière pour les professionnels de la voie publique. Dans son rapport déjà cité sur les policiers dans le métro parisien, Patricia Paperman montre toute l'importance de la perception des infimes ruptures de normalité du cours des choses. Elle montre que les policiers ont été formés à repérer, sur un mode presque éthologique, qui rappelle que les policiers sont plongés dans un monde qui, morphologiquement, est le même que les passants ordinaires, mais qui devient un Umwelt chargé d'alarmes, pour reprendre les termes de Goffman : «Donc, en général, on peut définir l'entourage ou Umwelt de l'individu comme étant la région à l'entour où peuvent apparaître les signes d'alarme auxquels il est sensible et où se localisent également les sources de ces alarmes $\|^{36}$. L'Umweltdu policier est un espace où l'agent ne repère que ceux qui lui émettent des alarmes précises, comme celles qui résultent de la peur... d'être contrôlés. Le " comme tout le monde » évoqué dans notre entretien est à ce titre convoqué pour montrer qu'il est citoyen ordinaire et qu'il ne relève pas de l'Umwelt policier. Contrôlé, il constate qu'il n'en est rien, en même temps qu'il est emmené ailleurs.

Reprenons notre problématique initiale là où nous l'avons laissée. Des populations, qui se définissent par une fréquentation constante de la rue et par une activité délictuelle, passée ou présente, intense, semblent faire l'objet d'un traitement à part. Ils sont séparés des autres, ôtés à l'espace public, maintenus dans des espaces ou sur des sentiers qui les prêtent au contrôle policier, à son tour instrument de repérage et de traitement de ces populations. La police, on le voit, dispose d'une faculté d'organisation minimale de l'établissement et de la circulation dans la ville. Mais elle détient aussi une compétence de sélection, une prérogative de définition des individus qu'elle soumet à ses stratégies de territorialisation. Cette faculté repose sur le sens intuitif des situations des policiers, qui interviennent lorsque les événements, les choses ou les gens qu'ils observent ne sont pas conformes à ce qui devrait être. Les policiers qualifient les populations qu'ils traitent par la territorialisation; ce qui ne manque pas de rappeler les mécanismes par lesquels, selon Schmitt, l'ennemi intérieur se voit désigné à son statut. A ceci près qu'il reste à montrer que les interactions dont nous venons de parler ne relèvent pas de simples échanges entre des catégories distinctes d'agents sociaux, mais que l'Etat est directement en jeu dans ces jeux-là; l'Etat qui ne satisfait sa prétention à la puissance politique que lorsqu'il définit avec succès son ennemi intérieur.

\section{Le ban}

Les entretiens que nous avons analysés témoignent des processus de socialisation par lesquels les marginaux et les policiers s'engagent dans une relation ancrée, mécanique de repérage, de sélection puis de traitement des premiers par les seconds. Il s'agit là en effet de processus de socialisation, en ce sens que les comportements face aux policiers, comportements qui motivent l'intervention de ces derniers, sont la conséquence de 
représentations sociales partagées à l'égard de la police, de la probabilité de son intervention, de la nature de son action. L'émotion, celle qui fragilise, comme nous l'avons dit plus haut, la valeur intrinsèque du témoignage, produit du comportement, et celui-ci, à son tour, définit bel et bien la probabilité de l'intervention policière, et l'enchâssement de cette dernière dans un protocole dont l'une des modalités est la mise au ban. Ce déterminisme situationnel, tant il dépend de l'agilité de la personne concernée à jouer des apparences, est aussi un déterminisme social, tant la capacité à jouer de l'apparence est induite par la capacité à pouvoir se retirer de l'espace public. Mais il est aussi, dans le cas qui nous intéresse, un déterminisme juridique, dans la mesure où l'action des policiers et notamment leurs prétentions à empêcher ou restreindre la liberté d'aller et venir sont soumises à la loi. C'est elle qu'il faut donc à présent examiner, gardant à l'esprit, bien sûr, que la superposition des compétences pratiques et des prérogatives juridiques est la mieux à même de tester la pertinence de l'hypothèse d'un ennemi intérieur.

\section{La loi et l'intervention policière}

Les stratégies de marginalisation dans l'espace urbain sont subies par des personnes qui sont fortement marginalisées dans l'espace social, au point que le territoire joue le rôle, en conséquence, de redondance du statut social aux yeux de qui est objet de ces stratégies : la marginalisation spatiale redouble la marginalité sociale/pénale. Quel est toutefois l'espace juridique dans lequel se situe la constitution de ces territoires policiers : comment le droit positif ou la jurisprudence considèrent ces territoires en marge?

On en a vu ainsi l'importance cruciale du contrôle d'identité, déclencheurs de l'action : c'est dans leur anticipation même que des comportements microsociologiques se convertissent en probabilité macrosociologique d'occurrence des contrôles. Depuis le fameux arrêt Friedel par lequel la Cour de cassation fixa en 1973 les règles générales en matière de contrôle d'identité sur la voie publique, les révisions successives du Code de procédure pénale ont en la matière été gouvernées par la reconnaissance, ou au contraire la restriction, de la part d'opportunité laissée aux policiers ${ }^{37}$. La dernière étape de ces conflits est fixée sous la forme de la loi du 10 août 1993, intégrée dans l'art. 78-2 du Code de procédure pénale: «L'identité d'une personne, quel que soit son comportement, peut être également contrôlée (...) pour prévenir une atteinte à l'ordre public, notamment à la sécurité des personnes et des biens ». Cette disposition marque une rupture, en ce que pour la première fois l'intervention policière préventive, non liée à un fait effectif et repérable, est détachée du comportement de la personne ${ }^{38}$. Le contrôle devient donc entièrement discrétionnaire. Les policiers ont ainsi, par cette révision du Code, latitude à définir qui relève de leur clientèle, qui ils retireront l'espace d'un instant du flux de ceux qui vont et viennent, et sur qui, éventuellement, ils feront pression pour quitter le quartier sur lequel ils effectuent le contrôle.

Cette disposition a suscité une "réserve d'interprétation » du Conseil constitutionnel, par laquelle, sans prononcer la non conformité du texte à la Constitution, le Conseil "enferme » la recevabilité de l'application de la proposition au respect de certaines dispositions : «considérant (...) que la pratique de contrôles d'identité généralisés et discrétionnaires serait incompatible avec le respect de la liberté individuelle, que, s'il est loisible au législateur de prévoir que le contrôle d'identité peut ne pas être lié[au]comportement[de lapersonne contrôlée], il demeure que l'autorité concernée[la 
police - NdA] doit justifier, dans tous les cas, des circonstances particulières établissant le risque d'atteinte à l'ordre public qui a motivé le contrôle, (...) ainsi il revient à l'autorité judiciaire, gardienne de la liberté individuelle de contrôler en particulier les conditions relatives à la légalité, à la réalité et à la pertinence des raisons ayant motivé les opérations de contrôle et de vérification de l'identité(... ${ }^{39}$ ». Convoquant de manière classique l'article 66 de la Constitution de 1958 sur le juge judiciaire fait gardien de la liberté individuelle, le Conseil indique que, en cas de contestation devant l'autorité judiciaire de la légalité de ce type de contrôles d'identité, les policiers doivent impérativement être en mesure de montrer par des éléments matériels probants que la personne contrôlée ou les circonstances pouvaient inviter à penser que l'ordre public, la sécurité des personnes et des biens pouvaient se trouver menacés, ce afin de ne pas voir le contrôle et les décisions incidentes (fouille, vérification, rétention administrative, etc.) cassées.

\section{La loi et ses conditions sociales d'effectivité}

C'est par cette décision que peut le mieux se comprendre le statut politique de ces territoires policiers. Il faut pour cela évoquer les conditions sociales de mobilisation des ressources judiciaires en vue de la contestation d'une décision policière aussi fluide et insignifiante (dans l'espace social ordinaire) qu'un contrôle d'identité. Imagine-t-on en effet un instant cette population, qui précisément se trouve le plus susceptible de se voir contrôlée, avoir recours à l'institution judiciaire pour contester un contrôle d'identité que bien souvent personne d'autre que les acteurs engagés dans l'interaction n'ont gardé en mémoire? L'entretien cité précédemment a montré que, en matière d'usage supposé abusif de la force, l'auteur de la plainte se distinguait par le fait qu'il ne relevait pas de la clientèle policière et témoignait donc devant l'autorité judiciaire de la double illégitimité dont il se trouvait victime : usage illégitime de la force, mais aussi assimilation illégitime de son apparence à un statut de marginal. La jurisprudence, par ce fait, est rare sur ce point ${ }^{40}$. Cette rareté témoigne, s'il le fallait, de la difficulté de l'accès à l'arène judiciaire de la part des populations concernées ${ }^{41}$.

Ainsi, le Conseil constitutionnel, par cette réserve d'interprétation, pose lui-même les conditions de nullité sociale de ses propres dispositions. Pour le dire autrement, le Conseil, en rendant le juge répressif seul juge de la police en matière de contrôles préventifs d'identité, conforte le caractère discrétionnaire de la décision policière et consolide du même coup l'espace social dans lequel les policiers peuvent asseoir leurs pratiques de territorialisation sur la foi du simple contrôle d'identité. Les territoires policiers se trouvent ainsi consolidés par la dépossession sociale des personnes visées et par l'interstice juridique particulièrement flou dans lequel s'inscrivent ces contrôles (entre police administrative et police judiciaire, où tout repose en dernière instance sur l'enfermement ineffectif prononcé par le Conseil constitutionnel). La preuve de cette consolidation trouve à lire dans la jurisprudence des tribunaux correctionnels éventuellement saisis, qui convoquent la marginalité de certaines zones urbaines pour les rendre légitimement plus propices aux contrôles ${ }^{42}$. Ils convertissent également en ressources légitimantes des contrôles, les comportements microsociologiques d'anticipation des personnes qui se trouvent sur certains quartiers ${ }^{43}$. Ce faisant, l'ordre juridique consolide le contrôle d'identité comme instrument de constitution des territoires policiers à l'égard de personnes que les policiers estiment relever de leurs 
compétences, et consolide également la dépossession sociale à la source de ces contrôles, ainsi que le régime d'impuissance dans lequel ces assujettis sont maintenus.

Il faut conclure en observant l'existence de trois espaces concordants, dont la première dimension est urbaine, la deuxième est sociale et la troisième est juridique. Dans leurs trois dimensions urbaine, sociale et juridique, ils ont en commun d'être des espaces marginaux, en ce que la police les sépare du reste de la ville, leurs attributs sociaux et pénaux les séparent du reste de la population et les instances judiciaires les confinent hors d'atteinte du juge judiciaire gardien des libertés individuelles. Il existe donc au sein de notre espace urbain ordinaire des territoires de ban, où des populations marginales se trouvent assujetties à la décision policière constituée par le droit comme décision dernière. $\mathrm{La}$ "pacification" des troubles ordinaires, la restauration ou la garantie de la " tranquillité, la sécurité et l'ordre » s'opèrent donc dans l'aménagement d'un espace particulier, constitué par la réunion de trois dimensions, géographiques, sociales et juridiques, chacune à la marge de son ordre propre. Tout invite à dire, finalement, que la loi concède aux policiers une liberté certaine à définir l'adversaire, celui qu'ils traitent, dans la ville, par la mise au ban; que cette mise au ban est aussi une mise hors la loi, du fait que la loi a elle-même rejeté le recours judiciaire contre cette mise hors la loi dans un horizon d'improbable social ; que l'État confère ainsi à ses agents le monopole de définir ceux par lesquels s'éprouve sa prétention à maintenir la tranquillité et l'ordre publics; et qu'en bout de course la réunion de ces trois appartenances (sociales, géographiques et juridiques) fait bien de celui qui la supporte un ennemi intérieur.

Si le constat est juste, force est de convenir qu'il n'est pas neuf : tout rappelle, dans ces modes de traitement des délinquants, des criminels et des fauteurs de trouble, les tactiques les plus anciennes de police. Il reste donc, de ce point de vue, à faire la preuve que l'inflation lexicale et matérielle des dispositifs de lutte contre l'ennemi intérieur signe une emprise renouvelée et accrue de l'État, de ses agents et de ses techniques, sur l'ordre quotidien. Pour en rester là sur un chapitre qui mériterait développement, celui de la permanence des modes de conservation de l'ordre, le domaine de validité sociologique de l'ennemi intérieur apparaît bien étroit.

Et puis, y a-t-il dans cette brève phénoménologie des techniques d'ordre signe de la validité du décisionnisme schmittien, point de départ de l'inquiétude de la sociologie critique des dispositifs sécuritaires? La réunion de ces trois marginalités fait-elle de celui qui la porte et la subit un ennemi intérieur? Les situations commandent la décision, les contingences sociales déterminent l'intervention, le sens ordinaire des situations des policiers fonde leur prétention à décider et agir. Et c'est la loi même qui, en posant a contrarioles conditions sociales de son efficacité, signe bien le caractère toujours contingent de la décision policière de mise au ban. Cette décision ne repose jamais, in fine, que sur le constat par les policiers de ce qui est déjà là, un trouble dans l'ordre social, une inadéquation entre ce qui est et ce qui devrait être. La loi, donc, ne concède leur puissance politique aux policiers que dès lors et pour autant que ces derniers ne visent que ceux que retienne leur impuissance sociale d'un recours devant le juge. La décision de mise au ban n'est donc pas pure, libre de toute limite et de toute contingence, pas plus qu'elle ne précède l'action, puisqu'elle se prend dans l'action; bref : cette décision de mise au ban n'a rien du caractère absolu et transcendant, préalable à l'instauration de l'ordre politique et condition de la stabilité de l'ordre social, comme l'exige la décision de Schmitt ${ }^{44}$. La police ne met pas au ban: elle n'y maintient jamais que ceux qui s'y trouvent déjà. Ce n'est pas l'ennemi intérieur qu'elle 
déclare, mais la délinquance qu'elle isole, sur cet archipel à ciel ouvert qui forme le topos manifestement imprenable de la pénalité moderne.

\section{NOTES}

1. Le Trosne, Mémoire sur les vagabonds et les mendiants, Soissons, 1764, p.4, cité in Wahnich S., L'errant, entre liberté et exclusion, Politix, 34, 1996, p. 43 et, avec le passage entre crochets, par Foucault, Surveiller et punir. Naissance de la prison, Paris, Gallimard, coll. "Bibliothèque des histoires ", 1975, p.79.

2. Jean-Jacques Rousseau, Contrat social, Livre II, chap.V., cité dans Foucault M., Surveiller et punir. Naissance de la prison, Paris, Gallimard, coll. « Bibliothèque des histoires », 1975, p.93.

3. Schmitt C., préface (1963) à la nouvelle édition de Der Begriff des Politischen, Berlin, Duncker und Humblot, 1965, p.11.

4. Ce sont là des choses connues, que rappellent le livre de Dewerpe A., Espion. Une anthropologie historique du secret d'État contemporain, Paris, Gallimard, coll. « Bibliothèque des histoires ", 1994 (notamment le chapitre 3, «La lumière de l'occulte »), en Allemagne Horn E., «Secret Intelligence. Zur Epistemologie der Nachrichtendienste » in Maresch R., Werber N. (dir.), Macht, Raum, Wissen, Frankfurt a Main, Suhrkamp 2001 (à paraître) ou en Grande-Bretagne l'article «Intelligence and counter-intelligence », in Encyclopaedia Britannica, 1991, 15e éd., pp. 717-724.

5. Nous renvoyons ici au texte de l'intervention de Didier Bigo à la Table ronde « Les figures de l'ennemi intérieur » du Congrès de l'Association française de science politique, tenu à Rennes du 28 septembre au 1er octobre 1999, et dont le présent texte est également issu. La contribution de Didier Bigo sera publiée ultérieurement dans Cultures \& Conflits. Nous profitons de cette incise pour remercier chaleureusement les participants de cette table ronde pour leurs commentaires et leurs critiques, ainsi que les lecteurs anonymes de Cultures \& Conflits.

6. Schmitt C., Der Begriff des Politischen, Berlin, Duncker und Humblot, 1965 (édition de 1932).

7. On remarquera bien sûr que Schmitt reprend ici les termes définissant l'ordre public dans la loi prussienne de 1795 sur les compétences de police, restée en usage jusque 1933, ou dans le code des communes françaises.

8. Nous reprenons la définition classique de Bittner E., The Functions of the Police in Modern Society, Cambridge, Odgeschlager, Grun et Hain Publishers, 1980 (1970) et « The capacity to use force as the core of the police rule ", traduit de l'américain et présenté par D. Monjardet dans Les Cahiers de la sécurité intérieure, 3, novembre 1990-janvier 1991, pp.224-235. Voir aussi le dossier que la revue Déviance et société consacre à Egon Bittner, dans son troisième numéro de 2001.

9. Marx G., Undercover Police Surveillance in America, Berkeley, 1988, p.219.

10. C'est là une des conclusions les plus fortes et les plus heuristiques de la sociologie de l'activité policière de pointe, que nous mentionnions récemment à propos de travaux récents (Jobard F., «Une police pure. La lutte contre la criminalité organisée vue par Norbert Pütter ", Cultures \& Conflits, 38-39, 2000, pp.241-254).

11. Fameux préfet de police qui succéda au début des années 1880 au préfet Andrieux, et qui était à l'époque plus connu sous le nom de "Camescasse-tête", en raison de la vigueur de sa politique répressive. Il est intéressant de constater que cette vigueur allait de pair avec un souci de professionnalisation du corps policier parisien, puisque le nom de Camescasse se trouve 
également associé à la première école de police de la Préfecture de police (je remercie Jean-Marc Berlière de la précision de ces détails).

12. Les filles de noces. Misère sexuelle et prostitution au XIXe siècle, Paris, Flammarion, coll. «Champs », 1982 (1978), p.157.

13. Le texte de Corbin précise que la loi ne pose que des cadres imprécis qui permettent la qualification de l'environnement. Le policier applique donc moins la loi qu'il ne procède à une évaluation des situations fondées sur la « compétence ordinaire de jugement ", définie comme la capacité à reconnaître les caractéristiques d'une situation et les qualités des protagonistes (voir Le petit B., Les formes de l'expérience. Une autre histoire sociale, Paris, Albin Michel, 1995, p.20).

14. Règlements de la police parisienne du 16 novembre 1843, puis du 15 octobre 1878.

15. Ce terme tente de traduire la notion américaine de " police property ", qui rend compte dans la sociologie interactionniste de la police des relations des policiers avec ceux qu'ils rencontrent le plus souvent et qu'ils s'emploient à maintenir sur des territoires déterminés (voir notamment Cray E., The Enemy in the streets, New York, Anchor, 1972 et Lee S., "Some structural aspects of police deviance », in Shearing $\mathrm{Cl}$., Stenning, $\mathrm{Ph}$ (dir.), Police Organizational Deviance, Toronto, Butterworth, 1981, pp.49-82).

16. Jobard F., L'usage de la force par la police. Sur quelques aspects de la mise en œuvre du monopole de la violence physique légitime par la police nationale dans la France contemporaine, Paris, Institut d'études politiques, 1998, à paraître en 2001 aux éditions La Découverte, coll. «Textes à l'appui ».

17. D'après les données du C.E.S.D.I.P publiées in Robert, Philippe et al., Les comptes du crime. Les délinquances en France et leurs mesures, Paris, L'Harmattan, 1994, p.114.

18. Ces chiffres sont à prendre avec précaution : ils sont obtenus sur déclaration spontanée des intéressés et non vérifiés. Source : Le Verlan/POHL, association présente au S.R.A.I.O.S.P dans le but de faciliter aux requérants l'accès à un logement.

19. Source : Le Verlan/POHL. Ces chiffres, ainsi que ceux qui suivent, sont établis d'après les dossiers judiciaires des requérants.

20. Français : $87,5 \%$; maghrébins : $7 \%$; Afrique noire : $1,25 \%$; autres : $3 \%$.

21. Goffman E., Stigmate, Paris, Minuit, coll. « Le sens commun », 1975.

22. Cette caractérisation des interwievés par leurs attributs de façade permet de bien comprendre les logiques aux principes des interactions policières (voir de ce point les « codified designations ", indicateurs d'appartenance à la clientèle policière autant que d'appartenance sociale, utilisées par la police de Toronto in Hartjen C., « Police-Citizen encoutersSocial order in interpersonal interaction ", Criminology, 10, 1972, pp.61-84.

23. Dulong R., Le témoin oculaire. Les conditions sociales de l'attestation personnelle, Paris, Editions de l'EHESS, 1998, pp.59-68.

24. Il n'est pas impossible que cette suspicion fût éveillée par les vendeurs de la pharmacie, rappelant ainsi la dernière partie de la citation de Corbin sur les rafles organisées en réponse à la demande publique locale.

25. Pour illustrer cette partie du travail de police judiciaire consacré à la lutte contre les infractions à la législation sur les stupéfiants, voir l'impressionnante enquête de Kreuzer A., Stock J., Drogen und PolizeiEine kriminologische Untersuchung polizeilicher Rechtsanwendung, Bonn, Forum Verlag Godesberg, 1996 ou bien l'enquête menée en France, plus restreinte, mais décisive sur la valeur d'usage de l'interpellation des petits trafiquants, de Barré M.-Det al., Le consommateur de produits illicites et l'enquête de police judiciaire, Paris, Centre d'études en sociologie du droit et des institutions pénales, étude $n^{\circ} 19$, mars 2000.

26. On retiendra ici ces qualificatifs de taille et de grandeur, dans la perspective de Châteauraynaud F., La faute professionnelle, Paris, Métaillé, 1997, qui a montré comment ces répertoires discursifs de classement s'articulent à des politiques de qualification et disqualification. On remarquera au passage que l'évaluation de sa propre grandeur dans 
l'économie des classements pénaux par la personne interrogée se renverse, dans sa propre stratégie discursive, en une attestation d'innocence à portée générale : preuve de l'imbrication des tactiques policières et des perceptions de soi.

27. Bargel J.-L., Théorie générale du droit, Paris, Dalloz, coll. « Méthodes du droit », 1985, p.152.

28. Decocq A., Montreuil J., Buisson, J., Le droit de la police, Paris, Litec, 1991, p.227.

29. Ces concentrations des marchés illicites ont été à Francfort, Hambourg, Stockholm ou Copenhague décidées par des processus de politiques publiques municipales ou régionales : cf. Jobard F., Fillieule O., "Action publique sous dépendance. Conditions et effets du changement de paradigme dans la lutte contre la délinquance associée à la drogue en Europe ", Revue française de science politique (Paris), 6, 49, décembre 1999, pp.805-835.

30. Goffman E., Asile. Etudes sur la condition sociale des malades mentaux, Paris, Minuit, coll. Le sens commun, 1968 (édition américaine : 1961).

31. «L'état de dérèglement est donc renforcé par ce fait que les passions sont moins disciplinées au moment même où elles avaient besoin d'une plus forte discipline », Durkheim É., Le suicide, Paris, P.U.F., coll. « Quadrige », 1986, p281. Dans son essai sur Marcel Mauss et les origines de la sociologie française, Bruno Karsenti définit l'anomie comme un ensemble de « dérèglements, où la société ne parvient pas, pour différentes causes, à exercer sur les individus le pouvoir normatif qui la caractérise essentiellement " (L'homme total. Anthropologie, sociologie et philosophie chez Marcel Mauss, Paris, P.U.F., coll. «Recherches théoriques», 1997). Cette dimension polysémique de l'anomie, qui embrasse la notion de maîtrise des désirs ( On ne sait plus ce qui est possible et ce qui ne l'est pas, ce qui est juste et ce qui est injuste, quelles sont les revendications et les espérances légitimes, quelles sont celles qui passent la mesure. Par suite, il n'est rien à quoi on ne prétende », Le suicide, pp280-281), de discipline individuelle, et de contrôle par la loi rencontre très certainement une fonction cardinale de l'action de la police, sur laquelle nous reviendrons dans la conclusion.

32. Goffman E., La mise en scène de la vie quotidienne, Paris, Minuit, coll. « Le sens commun », 1973 (1959), t.2, p.229.

33. L'approche théâtrale, au sens de Goffman, de l'interpellation du fraudeur par le contrôleur est parfaitement retracée in Dartevelle M., «Le travail du contrôleur ", Annales de la recherche urbaine, 57-58, 1993, pp.112-113. On retrouvera dans l'ouvrage de Isaac Joseph (La ville sans qualités, Paris, L'Aube, 1998) une sociologie attentive à la constitution de l'espace urbain comme agencements de situations et de compétences situationnelles, sociologie à laquelle notre contribution est fortement redevable. Voir aussi «Les compétences de rassemblement. Une ethnographie des lieux publics ", Enquête, 4, 1996, pp.107-122.

34. Dans les logiques pratiques de classement opérées par les policiers, on comprend que les variables d'habitude "secondaires" prennent le pas sur les indicateurs classiques de capital scolaire et capital économique). C'est pourquoi, rappelons-le, nous avons préféré mentionner ces critères seconds dans la présentation de nos entretiens, répondant ainsi au vœu de notre stratégie de recherche : comprendre ce qui se joue dans l'interaction policière, comprendre les ressorts de la décision policière et ses déterminants.

35. Siméant J., La cause des sans-papiers, Paris, Presses de Sciences Po, 1999, p322L'auteur montre ainsi comment les mobilisations des étrangers en situation irrégulière obligent d'abord à la constitution d'espaces affranchis de la possibilité d'intervention de la police : « la pratique de la grève de la faim dans les églises permet une protestation sans grand danger d'arrestation, déterritorialisant en quelque sorte un mouvement public de clandestins » [p.324, c'est l'auteur qui souligne].

36. Goffman E., La mise en scène de la vie quotidienne, op. cit., 1973 (1959), t.2, p.241.

37. Il n'est malheureusement pas possible, ici, de retracer les mille et un conflits entre les policiers et la Cour de cassation, la Cour de cassation et le législateur, le législateur et le Conseil constitutionnel autour de cette question. Les articles juridiques et policiers restent sur ce 
chapitre d'excellentes références. Voir Lambert L., "Les nouveaux contrôles d'identité ou le triomphe de l'absurde ", in Tribune du commissaire de police, $n^{\circ} 29$, déc1984, p4 et suiv. ; Marcus, «Essai d'identification du débat sur les contrôles d'identité », in Revue de science criminelle et de droit pénal comparé, 1985, p153 et suiv. ; Le Gunehec, « La loi du 24 août 1993Un rééquilibrage de la procédure pénale ", in J.C.P., 1993, I.3720, nº 22 et suiv. ; Mayer D., «Prévention ou répression en matière de contrôles d'identité. Une distinction trompeuse », Dalloz, 1993, chron., p272 ; Picard É., «Les contrôles d'identité au regard des droits fondamentaux. Des régimes inutilement hétéroclites ", in Revue française de droit administratif, 1994, p959 et suiv. ; Pradel J., " Observations brèves sur une loi à refaire (à propos de la loi du 4 août 1993)", in Dalloz 1993, chronp39; Matsopoulou H., Les enquêtes de police, Paris, L.G.D.J., coll» Bibliothèque des sciences criminelles », t.32, 1996.

38. Les dispositions françaises sont, sur ce point, en harmonie avec les dernières dispositions relatives aux «stop and search » au Royaume Uni (Criminal Justice and Public Order Act, 1994 : " in certain localities the police can stop and search in the absence of reasonnable suspicion ») et au Land de Berlin (la révision de l'Allgemeine Sicherheit und Ordnung Gesetz, adoptée par le Sénat en avril 1999 permet aux policiers de procéder, sur toute la ville et non plus sur la quinzaine de places réputées dangereuses, à des «verdachtsunabhängige Kontrolle » et d'interdire a priori de certaines zones des personnes particulières).

39. Décision Conseil constitutionnel n 93-323 DC du 5 août 1993 (loi n 93-992 du 10 août 1993).

40. L'un des rares cas est celui de Mme Madouni, Française mariée à un homme d'origine maghrébine, contrôlée le 15 décembre 1992 à 21 heures à la Station Nation. Le cas, compliqué de violences et insultes à caractère raciste, est allé jusqu'en Cour de cassation (cf. Tribunal de grande instance de Paris - décision du 30 avril 1993 ; appel de Mme Bonvalot, fonctionnaire de police ayant procédé au contrôle - décision CA Paris du 21 février 1994 ; arrêt de la Chambre criminelle de la Cour de cassation le 7 février 1995 et enfin jugement final de l'espèce par la Cour d'appel de Versailles le 15 décembre 1995). C'est précisément parce que Mme Madouni ne relève pas des territoires policiers qu'elle a, a contrario, disposé des ressources nécessaires à la mobilisation des instances judiciaires.

41. Il faudrait ici, pour être complet, évoquer la sociologie des «manœuvres de dissuasion » des plaignants en matière de comportement supposé illégitime de la part des policiers (cf. Jobard F., Les violences policières, Etat des travaux anglo-saxons, Paris, L'Harmattan, coll. "Logiques politiques », 1999, pp.192-200). Quoi qu'il en soit, on remarquera sans ironie aucune que lorsque Thierry di Manno, spécialiste des décisions interprétatives des juges constitutionnels français et italien, veut illustrer le cas du « degré zéro de l'influence des réserves d'interprétation », celui du "seuil nul de l'efficacité concrète des réserves", c'est l'exemple de la décision n ${ }^{\circ}$ 93-323 du Conseil constitutionnel qu'il convoque («L'influence des réserves d'interprétation », in Drago G., Francois B., Molfessis N., La légitimité de la jurisprudence du Conseil constitutionnel, Paris, Economica, 1999, pp.205-207).

42. Lorsqu'il est établi que 22 délits ont été établis dans le périmètre d'une avenue en 35 jours, les services de police sont bien fondés à effectuer des contrôles d'identité dans ces lieux dans le cadre de leur mission de protection de l'ordre public (Tribunal correctionnel Toulouse, in Bulletin d'information de la Cour de cassation, 1994, p.358).

43. Cf. Chbcrim, 23 mai 1995, sur la régularité du contrôle effectuée sur cette personne qui, présente sur un quartier à forte délinquance, a fait demi-tour et accéléré le pas en apercevant un véhicule de police.

44. Pour qui ce qui fait l'ennemi, c'est l'acte de Feinderklärung, cette déclaration par laquelle la communauté, se désignant un ennemi, accède à l'existence politique, au statut d'État (Der Begriff des Politischen, 1965, op. cit., pp45-54). 
INDEX

Mots-clés : ennemi, exclusion, Gendarmeries et maintien de l'ordre, police 